\section{Determinantes contextuais e individuais da utilização da triagem auditiva neonatal: Pesquisa Nacional de Saúde, 2013}

\author{
Contextual and individual determinants of use of \\ newborn hearing screening: the Brazilian National \\ Health Survey, 2013
} Determinantes contextuales e individuales en
la utilización del tamizaje auditivo neonatal:
Encuesta Nacional de Salud, 2013
Vanessa de Melo-Ferreira 1

Washington Leite Junger 1

Guilherme Loureiro Werneck 1

\title{
Resumo
}

O presente estudo avaliou o efeito de determinantes contextuais e individuais da utilização da triagem auditiva neonatal no Distrito Federal e capitais brasileiras. O modelo teórico conceitual proposto por Andersen er Davidson (2014) orientou as análises mediante modelagem logística multinível, com dados da Pesquisa Nacional de Saúde, 2013. A população observada $(n=585)$ é representativa de 230.112 duplas de mulheres/responsáveis acima de 18 anos e respectivas crianças menores de 2 anos de idade. No nível contextual (Distrito Federal e capitais), a utilização da triagem auditiva neonatal foi determinada pela proporção de indivíduos extremamente pobres (razão de chances $-R C=0,91$; intervalo de 95\% de confiança - IC95\%: 0,83-0,99) e pela cobertura mensal da triagem auditiva neonatal ( $R C=1,02$; IC95\%: 1,01-1,02). No nível individual, verificou-se maior uso de triagem auditiva neonatal na faixa etária entre 25 e 39 anos, em comparação às < 25 anos. A chance de utilização de triagem auditiva neonatal foi menor em respondentes com raçal cor da pele parda $(R C=0,47$; IC95\%: 0,26-0,83) em comparação com brancas. Quanto à escolaridade, verificou-se que possuir Ensino Superior completo quase triplicou a chance de uso de triagem auditiva neonatal, em contraste com Ensino Fundamental de estudo formal ( $R C=2,99$; IC95\%: 1,15-7,79). $O$ pré-natal predominantemente privado aumentou em 2,18 vezes a chance de utilizar a triagem auditiva neonatal, em comparação ao público $(R C=2,18$; IC95\%: 1,02-4,64). A aplicação efetiva de leis e políticas de saúde auditiva já existentes e a priorização de práticas de atenção básica e educação em saúde com foco em neonatos em maior vulnerabilidade, conforme as características identificadas nesse estudo, são iniciativas para ensejar um sistema de proteção social equânime.

Triagem Neonatal; Audição; Determinantes Sociais da Saúde; Modelos Epidemiológicos; Acesso aos Serviços de Saúde

\author{
Correspondência \\ V. Melo-Ferreira \\ Instituto de Medicina Social, Universidade do Estado do Rio de \\ Janeiro. \\ Rua São Francisco Xavier 524, bloco E, 7o andar, Rio de Janeiro, \\ RJ 20550-013, Brasil. \\ vanessademelo.ferreira@gmail.com \\ 1 Instituto de Medicina Social, Universidade do Estado do Rio de \\ Janeiro, Rio de Janeiro, Brasil.
}




\section{Introdução}

O primeiro ano de vida de uma criança constitui um período crítico para o desenvolvimento típico das habilidades auditivas e, por conseguinte, para a aquisição de linguagem. Essas conquistas são possíveis desde que ocorra integridade estrutural e fisiológica do sistema auditivo, estimulação ambiental adequada e maturação neurológica ${ }^{1}$. Consequentemente, devido a impactos no desenvolvimento global, rupturas prematuras nesses processos fazem com que a privação sensorial a estímulos auditivos na infância gere repercussões potencialmente mais devastadoras do que perdas auditivas com início na vida adulta 2.

A perda auditiva pode comprometer as relações interpessoais, promover dificuldades nos processos de aprendizagem ao longo da vida, levando ao baixo desempenho escolar, redução das opções profissionais futuras e forçosa dependência econômica, além de prejuízos no bem-estar psicossocial 3,4,5.

Se a perda auditiva é identificada com precocidade, famílias, sistemas de saúde e sociedade podem integrar-se para compreender as necessidades de cada criança com o objetivo de viabilizar ações pautadas no respeito às diversidades que estimulem próspero florescimento e desenvolvimento de suas potencialidades 5,6 .

Harris et al. 7 consideram que a perda auditiva no início da vida e seu impacto global sejam mais comuns em países em desenvolvimento, fazendo com que a carga das condições a ela relacionadas não seja homogeneamente distribuída no mundo 7,8. Essa carga excessiva da perda auditiva nos países em desenvolvimento decorre, precipuamente, do acesso limitado aos serviços de saúde perinatal, taxas mais elevadas de rubéola materna, sífilis e outras infecções, baixo peso ao nascer, asfixia neonatal, hiperbilirrubinemia severa e não tratada, administração de medicação ototóxica, consanguinidade e acesso restrito às vacinas $9,10,11,12,13,14$.

De acordo com a Organização Mundial da Saúde (OMS), 75\% das perdas auditivas em menores de 15 anos são evitáveis em países de baixa e média renda 6 . Assim, práticas de prevenção são imprescindíveis para a redução da carga das condições relacionadas à perda auditiva 5,8 .

Diferentes organizações ao redor do mundo têm estado alertas para a necessidade de identificação e intervenção dos agravos auditivos o mais rapidamente possível em todas as crianças que nascem. Tais estratégias são reconhecidas como programas de triagem auditiva neonatal universal 15,16,17.

No âmbito do Programa de Triagem Auditiva Neonatal Universal brasileiro, preconiza-se o uso de emissões otoacústicas evocadas (EOA) e/ou do potencial evocado auditivo de tronco encefálico (PEATE). Neonatos com baixo risco para o desenvolvimento de perda auditiva realizam uma segunda EOA em casos de falha. Já os neonatos de alto risco ou que falham na segunda tentativa de utilização da EOA, por sua vez, realizam até duas etapas do PEATE 18.

Nesse estudo, triagem auditiva neonatal será o termo utilizado para se referir às EOA, por ser um dos procedimentos padronizados de entrada nos programas de cuidado à saúde auditiva na infância, expressando, em linhas gerais, o primeiro uso do serviço de atenção à saúde auditiva neonatal.

Indica-se a triagem auditiva até o 1o mês de vida, diagnóstico audiológico até 3 meses e intervenção precoce até os 6 meses de idade nos casos confirmados 17. Índices superiores a 95\% de neonatos, tentando atingir 100\%, são recomendados como meta de cobertura para a triagem auditiva neonatal 16 .

Em 2010, a Lei no 12.30319 estabeleceu que a triagem auditiva neonatal é um direito de todas as crianças nascidas nos hospitais e maternidades do Brasil, devendo ser ofertada gratuitamente.

Em 2011, a triagem auditiva neonatal passou a fazer parte da Rede Cegonha 20, estratégia que inaugurou no âmbito do Sistema Único de Saúde (SUS), um novo modelo de atenção à saúde da mulher e de crianças de 0 a 2 anos de idade 21.

A utilização é uma das dimensões do acesso a serviços de saúde, podendo ser considerada como cerne dos sistemas de saúde 22,23. Neste sentido, um dos modelos explicativos mais aplicados na investigação de determinantes de acesso e utilização de serviços de saúde, é o modelo comportamental de Andersen 22,24,25.

$\mathrm{O}$ arcabouço teórico proposto estabelece que determinantes contextuais e individuais podem ser subdivididos em características predisponentes, capacitantes e de necessidade. As características predisponentes representam condições que fazem alguém ser mais propenso ao uso de um serviço específico. As características capacitantes incluem aspectos que favorecem ou impedem o uso dos serviços. Já as características de necessidade constituem condições que tanto leigos quanto profissionais 
de saúde reconhecem como exigentes de cuidados em saúde. Adicionalmente, incluem-se comportamentos de saúde e medidas de resultado 26 .

No Brasil, estudos epidemiológicos de base populacional sobre prevalência da perda auditiva congênita são inexistentes, e estimativas se baseiam, principalmente, em dados hospitalares. Evidenciando-se 2 a 2,3 casos em mil nascidos vivos 27,28,29.

Paschoal et al. 30 mediante análise espaço-temporal, atualizou as informações sobre cobertura da triagem auditiva neonatal para 161 regiões intermediárias de articulação urbana, entre 2008 e 2015. Houve evolução de 9,3\% para 37,2\% na cobertura da triagem auditiva neonatal no Brasil, evidenciando aumento das dimensões da estratégia, principalmente na Região Sul. Em 12 regiões, encontraram-se abrangência maior do que $95 \%$. Por outro lado, 28 regiões apresentaram cobertura de $0 \%$, sendo 27 destas concentradas nas regiões Norte e Nordeste.

A triagem auditiva neonatal passou a compor um dos cuidados neonatais indispensáveis no domínio da atenção básica, sublinhada como um direito de todas as crianças que nascem no Brasil. Quanto mais cedo se identifica casos de perda auditiva, mais efetivo tende a ser o encadeamento de atividades de intervenção que repercutem no desenvolvimento da linguagem, nas relações psicossociais e na qualidade de vida das crianças identificadas.

A despeito dos inegáveis avanços, ainda existe limitada utilização efetiva da estratégia no país, desvelando desigualdades de acesso ao programa brasileiro de saúde auditiva, que incidem, particularmente, na utilização da triagem auditiva neonatal 30,31,32.

Nesse contexto, pretendeu-se estimar o efeito de determinantes contextuais e individuais da utilização da triagem auditiva neonatal nas capitais brasileiras, com a finalidade de viabilizar ações que contribuam para o enfrentamento de iniquidades observadas no atendimento da população.

\section{Métodos}

Trata-se de um estudo transversal com dados da Pesquisa Nacional de Saúde (PNS), inquérito populacional de base domiciliar, desenvolvido pelo Instituto Brasileiro de Geografia e Estatística (IBGE) juntamente com o Ministério da Saúde realizado no ano de 2013 33. Como inovação, a PNS aborda aspectos relevantes em atenção à saúde da mulher, ao atendimento pré-natal e saúde de crianças de 0 a 2 anos de idade, presentes na Rede Cegonha 34.

A amostra estudada na PNS foi representativa para moradores de domicílios particulares, das zonas urbana e rural, das cinco grandes regiões geográficas, estados, regiões metropolitanas e capitais dos estados brasileiros. As unidades de amostragem primária (UPA) foram definidas por setores censitários ou por um conjunto desses, com pelo menos 60 domicílios. A amostragem obedeceu três estágios: as UPA foram selecionadas com probabilidade proporcional ao tamanho considerando o número de domicílios em cada unidade; os domicílios foram selecionados por uma amostragem aleatória simples em cada UPA selecionada; e indivíduos com 18 anos de idade ou mais foram selecionados por amostragem aleatória simples dentre os adultos do domicílio para responder um questionário específico 33.

A população observada $(n=585)$ é representativa de 230.112 duplas de pessoas identificadas pelo inquérito como mulheres/responsáveis acima de 18 anos e crianças de menos de 2 anos de idade, residentes nas capitais brasileiras e no Distrito Federal. Os critérios de inclusão foram: (1) crianças com data de nascimento entre 28 de julho de 2011 e 21 de maio de 2013; (2) residentes com último parto ocorrido em igual período; (3) participantes identificadas como mães/responsáveis de criança de menos de 2 anos e que responderam "sim" ou "não" à pergunta: "Foi realizado o teste da orelhinha?" (módulo L) - a opção "não sabe" foi excluída (n = 9); (4) pessoas com respostas "sim" ou "não" à pergunta: "Na última vez que a senhora esteve grávida, a senhora fez pré-natal?” (módulo S) 35.

O desfecho foi classificado em "triagem auditiva neonatal utilizada" e "triagem auditiva neonatal não utilizada”. As variáveis individuais representam características de saúde da mulher/responsável em consonância ao modelo teórico 26 dividindo-se em: (1) predisponentes: faixa etária; cor/raça; vive com companheiro(a); escolaridade; atividade remunerada; número de partos; (2) capacitantes: domicílio cadastrado em unidade de saúde da família; possuir plano de saúde; localização da maioria das consultas pré-natais; localização do parto; (3) necessidade: dificuldade auditiva; (4) comportamentos 
em saúde: número de consultas pré-natais; alguma orientação sobre sinais de risco na gravidez; exame de sangue para sífilis; pedido de exame de sífilis para parceiro; solicitação de teste para HIV 26.

A frequência de dados faltantes foi mínima e concentrada em seis variáveis. Em cinco destas, a proporção de não resposta variou de 1,42\% a 3,98\%. No entanto, chama a atenção, que especificamente para a variável solicitação de teste para triagem da infecção por sífilis do "parceiro", tal proporção tenha sido 99,73\% (dados não mostrados em tabela). Desta forma, esta última variável não foi incluída nas análises.

As variáveis contextuais em nível de capital e Distrito Federal advêm de sistemas de informação (Sistema de Informações sobre Nascidos Vivos - SINASC); Produção ambulatorial do SUS; Sistema de Informações Hospitalares - SIH; Sistema de Informações sobre Mortalidade - SIM e Sistema de Informação de Agravos de Notificação - SINAN) e classificam-se em: (1) Predisponentes: proporção de extrema pobreza e de pobreza (proporção de pessoas com renda domiciliar per capita igual ou inferior a R\$ 70 e R\$ 140 mensais, em agosto de 2010, respectivamente); Índice de Desenvolvimento Humano Municipal (IDH-M) nas dimensões renda, longevidade e educação 36; (2) Capacitantes: cobertura mensal de nascidos vivos com 7 ou mais consultas pré-natais; cobertura mensal da triagem auditiva neonatal; cobertura mensal de testes para sífilis em gestantes; proporção mensal de óbitos investigados em mulheres em idade fértil; proporção mensal de óbitos infantis investigados; (3) De necessidade: taxa mensal de mortalidade infantil e a taxa de incidência mensal de sífilis congênita confirmada em menores de 1 ano 37.

A análise descritiva das variáveis independentes foi realizada de acordo com a distribuição proporcional do desfecho. Utilizou-se teste binomial exato para a construção de intervalo de $95 \%$ de confiança (IC95\%). Diante da natureza hierárquica dos dados foi utilizada a análise de regressão logística multinível em dois níveis com intercepto aleatório.

Em amostras complexas, a probabilidade de seleção ao nível dos conglomerados é diferente da probabilidade de seleção de participantes 38 . Os pesos provenientes deste tipo de delineamento são pesos de amostragem e não pesos de frequência, cujo uso é tradicional em análises hierárquicas 39 . A inobservância dos pesos de amostragem pode gerar estimativas viesadas dos parâmetros, logo, procedeu-se o escalonamento dos pesos de amostragem apenas para os dados do primeiro nível ${ }^{39}$. Pois o escalonamento de pesos no segundo nível, não costuma influenciar as estimativas de parâmetros e respectivos erros padrão 38 .

Como a amostra estudada apresenta estratos de tamanho pequeno sem o plano amostral, aplicouse método proposto por Asparouhov 38 e Carle 40:

$$
w_{i j}^{*}=w_{i j}\left(\frac{n_{j}}{\sum_{i} w_{i j}}\right)
$$

onde, $w_{i j}^{*}$ representa o peso escalonado para o indivíduo $i$ na capital $j ; w_{i j}$ simboliza o peso não escalonado para o indivíduo $i$ na capital $j$; e $n_{j}$ é o número de unidades amostrais na capital $j$. A variável de estrato foi utilizada para indicar a estrutura de agrupamento, enquanto a variável de peso do domicílio com correção de não entrevista sem calibração pela projeção de população, constituiu o peso de amostragem individual 38,39,40.

Para respeitar as relações do arcabouço teórico, a inserção de variáveis estruturou-se em 12 modelos: modelos predisponentes, capacitantes, de necessidade e comportamentos de saúde. Em cada um destes, apenas as variáveis que atingiram valor de $\mathrm{p}<0,20$ mantiveram-se, não para reestimativa dos coeficientes, mas para sofrerem ajuste das estruturas subsequentes. As medidas de efeito foram mensuradas por razões de chances (RC) e a estatística de Wald foi utilizada para apreciar a significância estatística das variáveis independentes com IC95\%, via procedimento de máxima verossimilhança ${ }^{41}$.

Obteve-se o fator de inflação de variância para testar a multicolinearidade das variáveis 42 e teste de razão de verossimilhança para a comparação entre modelos. Para analisar a qualidade do ajuste, foram obtidos gráficos diagnósticos de resíduos e teste externo binomial exato do pacote DHARMa 43. O coeficiente de partição de variância (CPV) expressa a proporção da variância residual total atribuível ao segundo nível, de sorte que a variância do primeiro nível se modifica em função da média das variáveis explicativas 44 .

As análises foram processadas pelo software R versão 3.6.1 (http://www.r-project.org) e disponíveis no Repositório TAN na plataforma Github 45. 
A PNS 2013 foi aprovada pela Comissão Nacional de Ética em Pesquisa, do Conselho Nacional de Saúde (protocolo no 10853812.7.0000.0008). Os dados são públicos e desidentificados para garantir a privacidade dos participantes 35 .

\section{Resultados}

A prevalência da utilização da triagem auditiva neonatal, nas capitais brasileiras, foi de 78,42\% (IC95\%: 74,00-82,85). A Tabela 1 mostra que a prevalência do uso da triagem auditiva neonatal modificou-se conforme características específicas de mães/responsáveis. Apesar de não constituir o resultado mais elevado, destaca-se a prevalência de 78,27\% (IC95\%: 72,71-82,97) relativo ao subgrupo que relatou receber alguma orientação acerca de sinais de risco na gravidez. Por outro lado, dentre as crianças usuárias, não houve relatos de responsáveis com deficiência auditiva.

\section{Tabela 1}

Prevalência de utilização de triagem auditiva neonatal segundo características individuais da pessoa identificada como mãe ou responsável da criança de menos de 2 anos. Capitais e Distrito Federal, Brasil, 2013.

\begin{tabular}{|c|c|c|c|}
\hline & $\mathbf{n}$ & Prevalência (\%) & IC95\% \\
\hline Utilização da triagem auditiva neonatal & 180.462 & 78,42 & $74,00-82,85$ \\
\hline \multicolumn{4}{|l|}{ Características individuais } \\
\hline \multicolumn{4}{|l|}{ Predisponentes demográficas } \\
\hline \multicolumn{4}{|l|}{ Faixa etária (anos) } \\
\hline $18-24$ & 41.445 & 68,21 & $58,19-76,79$ \\
\hline $25-29$ & 46.106 & 77,67 & $67,10-85,58$ \\
\hline $30-34$ & 53.634 & 82,94 & $73,06-89,71$ \\
\hline $35-39$ & 30.663 & 86,94 & $71,06-94,75$ \\
\hline $40-44$ & 8.259 & 92,12 & $70,96-98,25$ \\
\hline $45-49$ & 354 & 32,48 & $2,92-88,49$ \\
\hline \multicolumn{4}{|l|}{ Raça/cor } \\
\hline Branca & 81.715 & 87,76 & $78,97-93,19$ \\
\hline Preta & 17.511 & 78,13 & $62,06-88,64$ \\
\hline Parda & 79.311 & 71,47 & $64,44-77,59$ \\
\hline Amarela ou indígena & 1.926 & 53,25 & $17,77-85,73$ \\
\hline \multicolumn{4}{|l|}{ Vive com companheiro(a) } \\
\hline Não & 35.862 & 79,10 & $68,30-86,93$ \\
\hline Sim & 144.601 & 78,26 & $72,62-83,01$ \\
\hline \multicolumn{4}{|l|}{ Predisponentes sociais } \\
\hline \multicolumn{4}{|l|}{ Escolaridade } \\
\hline Ensino Fundamental incompleto & 18.449 & 56,53 & $41,10-70,79$ \\
\hline Ensino Fundamental completo e Médio incompleto & 37.184 & 71,27 & $60,58-80,02$ \\
\hline Ensino Médio completo e Superior incompleto & 84.241 & 81,82 & $74,48-87,4$ \\
\hline Superior completo & 40.589 & 95,87 & $91,19-98,11$ \\
\hline \multicolumn{4}{|l|}{ Número de partos } \\
\hline 1 & 78.774 & 82,25 & $74,16-88,21$ \\
\hline 2 & 55.593 & 75,86 & $67,39-82,70$ \\
\hline 3 ou mais & 46.096 & 75,50 & $64,43-83,98$ \\
\hline
\end{tabular}

(continua) 
Tabela 1 (continuação)

\begin{tabular}{|c|c|c|c|}
\hline & $\mathbf{n}$ & Prevalência (\%) & IC95\% \\
\hline \multicolumn{4}{|l|}{ Capacitantes de financiamento } \\
\hline \multicolumn{4}{|l|}{ Trabalho ou estágio } \\
\hline Não & 98.598 & 72,73 & $65,57-78,89$ \\
\hline Sim & 81.864 & 86,58 & $79,92-91,28$ \\
\hline \multicolumn{4}{|c|}{ Acesso à unidade saúde da família } \\
\hline Não ou não sabe & 99.323 & 80,09 & $73,90-85,11$ \\
\hline Sim & 81.139 & 76,48 & $68,17-83,15$ \\
\hline \multicolumn{4}{|l|}{ Possui plano de saúde } \\
\hline Não & 100.966 & 68,90 & $62,35-74,78$ \\
\hline Sim & 79.496 & 95,12 & $88,35-98,04$ \\
\hline \multicolumn{4}{|l|}{ Capacitantes de organização } \\
\hline \multicolumn{4}{|l|}{ Local do pré-natal } \\
\hline Público & 94.863 & 68,90 & $61,63-75,35$ \\
\hline Privado & 83.039 & 94,05 & $88,71-96,95$ \\
\hline \multicolumn{4}{|l|}{ Local do último parto } \\
\hline Hospital ou maternidade & 179.297 & 79,09 & $74,48-83,06$ \\
\hline Outros & 1.165 & 34,11 & $6,73-78,79$ \\
\hline \multicolumn{4}{|l|}{ Comportamentos de saúde } \\
\hline \multicolumn{4}{|c|}{ Número de consultas de pré-natal } \\
\hline Menos de 7 & 45.100 & 66,63 & $56,50-75,43$ \\
\hline Mais de 7 & 132.802 & 83,90 & $78,68-88,04$ \\
\hline \multicolumn{4}{|l|}{ Processo de cuidados no pré-natal } \\
\hline \multicolumn{4}{|c|}{ Orientações sobre sinais de risco na gravidez } \\
\hline Não & 39.242 & 80,39 & $68,46-88,57$ \\
\hline Sim & 138.660 & 78,27 & $72,71-82,97$ \\
\hline \multicolumn{4}{|l|}{ Realizou teste para sífilis } \\
\hline Não ou não sabe & 33.985 & 75,09 & $62,74-84,37$ \\
\hline Sim & 139.292 & 79,83 & $74,36-84,38$ \\
\hline \multicolumn{4}{|l|}{ Foi solicitado teste de HIV } \\
\hline Não ou não sabe & 8.673 & 74,58 & $49,95-89,61$ \\
\hline Sim & 169.229 & 78,96 & $73,98-83,20$ \\
\hline
\end{tabular}

IC95\%: intervalo de 95\% de confiança.

As estimativas das associações entre a utilização da triagem auditiva neonatal e os diferentes determinantes contextuais e individuais são apresentadas na Tabela 2.

Em relação à característica predisponente de contexto, a chance de utilização da triagem auditiva neonatal foi reduzida em $9 \%$ a cada incremento de 1 unidade percentual na proporção de extrema pobreza $(\mathrm{RC}=0,91$; IC95\%: 0,83-0,99).

Quanto às variáveis predisponentes individuais, a faixa etária apresentou um gradiente importante. Crianças cujas mães/responsáveis tinham de 25 a 39 anos apresentaram chances mais elevadas de utilizar a triagem auditiva neonatal, quando comparadas a faixa etária de 18 a 24 anos. Sendo a chance de uso 2,82 vezes maior para responsáveis de 35 a 39 anos ( $\mathrm{RC}=2,82$; IC95\%: 1,17-6,80).

Os filhos de pessoas pardas tiveram $53 \%$ menor chance de utilizar a triagem auditiva neonatal, em comparação à participantes que se autodeclararam brancas ( $\mathrm{RC}=0,47$; IC95\%: 0,26-0,83).

Verifica-se ainda que a chance de realizar a triagem foi 2,99 vezes maior em crianças cuja mãe/ responsável referiu Ensino Superior, em relação àquelas com Ensino Fundamental incompleto de escolaridade ( $\mathrm{RC}=2,99$; IC95\%: 1,15-7,79). 
Tabela 2

Modelagem logística multinível de características associadas à utilização da triagem auditiva neonatal sem escalonamento e com escalonamento de pesos amostrais de Asparouhov 38 e Carle 40. Capitais e Distrito Federal, Brasil, 2013.

\begin{tabular}{|c|c|c|c|c|}
\hline & \multicolumn{2}{|c|}{$\begin{array}{l}\text { Sem escalonamento de pesos } \\
\text { amostrais }\end{array}$} & \multicolumn{2}{|c|}{$\begin{array}{l}\text { Com escalonamento de pesos } \\
\text { amostrais }\end{array}$} \\
\hline & RC (IC95\%) & Valor de p & RC (IC95\%) & Valor de $p$ \\
\hline \multicolumn{5}{|l|}{ Variáveis predisponentes } \\
\hline Proporção de extrema pobreza & $0,93(0,85-1,02)$ & 0,103 & $0,91(0,83-0,99)$ & 0,037 \\
\hline \multicolumn{5}{|l|}{ Faixa etária (anos) [referência: 18-24] } \\
\hline $25-29$ & $1,62(0,90-2,92)$ & 0,105 & $1,88(1,04-3,41)$ & 0,038 \\
\hline $30-34$ & $2,00(1,04-3,87)$ & 0,038 & $2,09(1,08-4,02)$ & 0,028 \\
\hline $35-39$ & $2,51(1,05-6,00)$ & 0,039 & $2,82(1,17-6,80)$ & 0,021 \\
\hline $40-44$ & $1,80(0,44-7,41)$ & 0,418 & $1,83(0,44-7,64)$ & 0,410 \\
\hline $45-49$ & $0,56(0,03-11,28)$ & 0,708 & $0,86(0,04-17,67)$ & 0,922 \\
\hline \multicolumn{5}{|l|}{ Raça/cor [referência: branca] } \\
\hline Preta & $0,77(0,32-1,82)$ & 0,545 & $0,83(0,34-1,99)$ & 0,672 \\
\hline Amarela ou indígena & $0,78(0,18-3,38)$ & 0,741 & $0,81(0,18-3,55)$ & 0,777 \\
\hline Parda & $0,50(0,28-0,88)$ & 0,017 & $0,47(0,26-0,83)$ & 0,010 \\
\hline \multicolumn{5}{|l|}{ Vive com companheiro(a) [referência: não] } \\
\hline Sim & $0,73(0,43-1,25)$ & 0,256 & $0,68(0,39-1,17)$ & 0,161 \\
\hline \multicolumn{5}{|l|}{ Escolaridade [referência: Ensino Fundamental incompleto] } \\
\hline Ensino Fundamental completo e Médio incompleto & $1,52(0,8-2,89)$ & 0,200 & $1,47(0,77-2,82)$ & 0,247 \\
\hline Ensino Médio completo e Superior incompleto & $2,02(1,07-3,8)$ & 0,029 & $1,87(0,99-3,54)$ & 0,055 \\
\hline Superior completo & $2,83(1,11-7,2)$ & 0,029 & $2,99(1,15-7,79)$ & 0,025 \\
\hline \multicolumn{5}{|l|}{ Número de partos [referência: 1] } \\
\hline 2 & $0,63(0,37-1,07)$ & 0,088 & $0,61(0,36-1,04)$ & 0,071 \\
\hline 3 ou mais & $0,85(0,44-1,63)$ & 0,617 & $0,80(0,41-1,56)$ & 0,513 \\
\hline \multicolumn{5}{|l|}{ Variáveis capacitantes * } \\
\hline Cobertura de nascidos vivos com 7 ou mais consultas pré-natal & $1,02(1,00-1,04)$ & 0,047 & $1,02(1,00-1,04)$ & 0,080 \\
\hline Cobertura da triagem auditiva neonatal & $1,01(1,01-1,02)$ & $<0,001$ & $1,02(1,01-1,02)$ & $<0,001$ \\
\hline Cobertura de testes para sífilis em gestantes & $1,00(1,00-1,00)$ & 0,504 & $1,00(1,00-1,00)$ & 0,549 \\
\hline Proporção de óbitos em mulheres em idade fértil & $1,00(0,98-1,02)$ & 0,718 & $0,99(0,98-1,01)$ & 0,579 \\
\hline \multicolumn{5}{|l|}{ Trabalho ou estágio [referência: não] } \\
\hline Sim & $1,34(0,80-2,25)$ & 0,266 & $1,30(0,77-2,19)$ & 0,322 \\
\hline \multicolumn{5}{|l|}{ Acesso à unidade saúde da família [referência: não/não sabe] } \\
\hline Sim & $1,18(0,72-1,93)$ & 0,506 & $1,22(0,74-1,99)$ & 0,434 \\
\hline \multicolumn{5}{|l|}{ Possui plano de saúde [referência: não] } \\
\hline Sim & $2,36(1,01-5,52)$ & 0,049 & $2,34(0,98-5,60)$ & 0,056 \\
\hline \multicolumn{5}{|l|}{ Local do pré-natal [referência: público] } \\
\hline Privado & $1,86(0,90-3,88)$ & 0,096 & $2,18(1,02-4,64)$ & 0,044 \\
\hline \multicolumn{5}{|l|}{ Local do parto [referência: hospital/maternidade] } \\
\hline Outros & $1,15(0,18-7,34)$ & 0,879 & $1,04(0,13-8,48)$ & 0,973 \\
\hline \multicolumn{5}{|l|}{ Variáveis de necessidade ** } \\
\hline Taxa de mortalidade infantil & $1,00(0,95-1,06)$ & 0,882 & $1,01(0,95-1,07)$ & 0,815 \\
\hline Taxa de incidência de sífilis congênita (<1 ano) & $1,02(0,99-1,06)$ & 0,205 & $1,02(0,98-1,06)$ & 0,258 \\
\hline
\end{tabular}

(continua) 


\begin{tabular}{|c|c|c|c|c|}
\hline & \multicolumn{2}{|c|}{$\begin{array}{l}\text { Sem escalonamento de pesos } \\
\text { amostrais }\end{array}$} & \multicolumn{2}{|c|}{$\begin{array}{l}\text { Com escalonamento de pesos } \\
\text { amostrais }\end{array}$} \\
\hline & RC (IC95\%) & Valor de $p$ & RC (IC95\%) & Valor de $\mathrm{p}$ \\
\hline \multicolumn{5}{|c|}{ Variáveis de comportamentos de saúde ** } \\
\hline \multicolumn{5}{|c|}{ Número de consultas de pré-natal [referência: menos de 7] } \\
\hline Mais de 7 & $1,58(0,97-2,57)$ & 0,065 & $1,61(0,98-2,63)$ & 0,058 \\
\hline \multicolumn{5}{|c|}{ Orientações/sinais de risco na gravidez [referência: não] } \\
\hline Sim & $0,87(0,49-1,53)$ & 0,624 & $0,90(0,50-1,6)$ & 0,712 \\
\hline \multicolumn{5}{|c|}{ Realizou teste para sífilis [referência: não/não sabe] } \\
\hline Sim & $1,02(0,57-1,81)$ & 0,954 & $0,98(0,54-1,77)$ & 0,938 \\
\hline \multicolumn{5}{|c|}{ Foi solicitado teste de HIV [referência: não/não sabe] } \\
\hline Sim & $0,97(0,33-2,81)$ & 0,949 & $0,90(0,30-2,75)$ & 0,857 \\
\hline
\end{tabular}

IC95\%: intervalo de 95\% de confiança; RC: razão de chance.

* Ajustado por proporção de extrema pobreza, faixa etária, raça/cor, vive com companheiro(a), escolaridade, número de partos;

** Ajustado por proporção de extrema pobreza, faixa etária, raça/cor, vive com companheiro(a), escolaridade, número de partos, cobertura de nascidos vivos com 7 ou mais consultas pré-natal, cobertura da triagem auditiva neonatal, posse de plano de saúde, localização do pré-natal.

Quanto aos determinantes capacitantes, a chance de uso da triagem auditiva neonatal aumentou $2 \%$ a cada 1 unidade percentual da cobertura mensal da triagem auditiva neonatal ( $\mathrm{RC}=1,02$; IC95\%: 1,01-1,02). Concomitantemente, observou-se que o pré-natal predominantemente privado aumentou 2,18 vezes a chance de utilizar a triagem auditiva neonatal, em contraste ao pré-natal preponderantemente público ( $\mathrm{RC}=2,18$; IC95\%: 1,02-4,64).

Os modelos de necessidade e comportamentos de saúde não apresentaram associações estatisticamente significativas após o ajuste para variáveis predisponentes e capacitantes.

O CPV sem variáveis explicativas indicou que as diferenças entre as capitais explicaram 22,76\% da variação encontrada na chance de utilização da triagem auditiva neonatal. Por outro lado, os CPVs dos modelos com variáveis explicativas, mostraram que a variabilidade diminuiu globalmente - indicando que as variáveis contextuais e individuais explicaram parcela expressiva da variação do uso da triagem auditiva neonatal entre as capitais brasileiras.

\section{Discussão}

O presente estudo utilizou o arcabouço teórico-metodológico proposto por Andersen \& Davidson 26 para avaliar o efeito dos determinantes contextuais e individuais predisponentes, capacitantes, de necessidade e comportamentos de saúde na utilização da triagem auditiva neonatal em crianças que residem nas capitais brasileiras.

Dentre variáveis predisponentes, verificou-se que a utilização da triagem auditiva neonatal foi determinada por características sociodemográficas. Na dimensão contextual, destacou-se a proporção de extrema pobreza. Já na perspectiva individual, observou-se contribuição da faixa etária, raça/ cor e escolaridade.

Dentre os aspectos capacitantes avaliados, a cobertura da triagem auditiva neonatal foi o indicador mais fortemente associado com sua realização, expressão da materialidade da política pública de saúde. Por outro lado, quando o local de realização da maioria das consultas pré-natais foi privado, a utilização da triagem auditiva neonatal também aumentou.

Nenhuma variável de necessidade ou de comportamentos de saúde foi significativamente associada com uso da triagem auditiva neonatal na população estudada.

Assim, a tessitura demográfica e o financiamento destinado ao serviço de saúde auditiva se combinaram com características individuais da mãe/responsável da criança determinando a utilização da triagem auditiva neonatal nas capitais brasileiras, reiterando-se a existência de disparidades regionais no acesso ao serviço 30,31,32. 
A despeito das peculiaridades da população investigada, o contexto local de extrema pobreza subverteu a lógica do acesso equânime. Características contextuais potencialmente influenciam comportamentos de saúde 26. A participação da dimensão predisponente contextual demográfica sinaliza que capitais povoadas com proporções acentuadas de pessoas extremamente pobres necessitariam dispor de composições diferenciadas de serviços. Especialmente, quanto à disponibilidade de serviços de atenção básica, dado que parte da população residente (sobre)vive em condições precárias.

Malheiros \& Cavalcanti 46 relatam importante recorte da realidade brasileira ao caracterizarem cinco programas de triagem auditiva neonatal de João Pessoa, Paraíba, Brasil, em 2014. Era prevista a contribuição de gestores locais, mas nenhum deles respondeu os questionários propostos. As autoras apontaram que o fonoaudiólogo era o único profissional envolvido nos programas e que somente em uma maternidade havia apoio de equipe de serviço social para auxiliar no diagnóstico audiológico. Em todas as maternidades os sistemas de gerenciamento de dados eram realizados manualmente. Em síntese, o serviço oferecido era pautado por participação insuficiente dos gestores, ausência de equipes multiprofissionais e carência de recursos ${ }^{46}$. Este breve panorama favorece a percepção de que residir em localidades com menor desenvolvimento propicia rupturas mais acentuadas no acesso aos sistemas de saúde auditiva 5,7,8.

Variáveis contextuais capacitantes expressam o grau de financiamento à serviços de atenção à saúde, indicando a possibilidade de que maiores investimentos em favor da disponibilidade de programas de triagem auditiva neonatal universal no âmbito do SUS reverberem em maior acesso de crianças de baixa renda, ou não asseguradas por planos de saúde. Desse modo, a oferta do serviço, retratada pela cobertura da triagem auditiva neonatal, foi decisiva para que a utilização ocorresse, contribuindo para mitigar iniquidades de acesso.

Quanto a faixa etária, estudos anteriores mostraram que mulheres de 20 a 35 anos foram mais frequentes dentre as mães/responsáveis de usuários da triagem auditiva neonatal 47,48 . De fato, os resultados aqui apresentados mostram que a chance de uso foi maior em crianças com mãe/responsável entre 25 e 39 anos, em contraste com a faixa etária referência de 18 e 24 anos.

Não foram encontrados estudos nacionais anteriores que relatassem raça/cor da pele como fator associado à utilização da triagem auditiva neonatal 49. A literatura indica que características étnico-raciais estão associadas tanto ao atraso no diagnóstico audiológico, quanto ao processo de (re)habilitação da perda auditiva na infância ${ }^{50}$. Há ainda evidências de que crianças afro-americanas experienciam maior probabilidade de perda no seguimento dos cuidados em saúde, mesmo após o diagnóstico de perda auditiva 51. Paralelamente, Leal et al. 52 apuraram que mulheres pretas e pardas alcançaram piores níveis de satisfação quanto à assistência ao parto, pré-natal e atendimento ao recém-nascido, no Município do Rio de Janeiro, entre 1999 e 2001.

Pessoas autorreferidas como pardas compuseram a maioria da população total estudada $(48,22 \%$, $\mathrm{n}=110.968)$. De modo iníquo, seus filhos sofreram as maiores dificuldades em utilizar a triagem auditiva neonatal. Indicativo que reitera o resultado da histórica discriminação racial e da exclusão social impingidas a esta população pelo racismo, que em suas amplas ramificações, opera restringindo o acesso aos serviços de saúde individual 53,54.

Com relação à escolaridade, quanto maior o nível educacional referido, maior foi a chance de uso da triagem auditiva neonatal. Sobretudo para o Ensino Superior completo, estrato para a qual a prevalência de utilização foi particularmente elevada (95,87\%).

Ravi et al. 55 demonstraram por meio de revisão sistemática que disparidades educacionais e falta de informações dos responsáveis foram frequentemente associados às altas perdas de seguimento em programas de triagem auditiva neonatal. Longas distâncias até os serviços, restrições com relação ao trabalho dos responsáveis pela criança, atitudes desfavoráveis e a competição com outras necessidades de saúde também foram consideradas relevantes. Dentre as estratégias efetivas destacaram-se sistemas de gerenciamento de banco de dados, maiores níveis de escolaridade dos responsáveis, implementação de lembretes telefônicos, equipes multidisciplinares e flexibilidade no agendamento de consultas.

Verificou-se que apesar de ser um procedimento legalmente universal, na prática, o uso da triagem auditiva neonatal foi condicionado por características socioeconômicas individuais. É plausível que mulheres/responsáveis com situação socioeconômica mais privilegiada puderam arcar com as despesas de um atendimento pré-natal majoritariamente privado, obtendo maior regularidade no 
acompanhamento da gestação, proporcionando a seus filhos, chances mais elevadas de usufruir da triagem auditiva neonatal.

É notório que a imagem sociocultural da mulher segue ocupando evidente exclusividade perante a linha de cuidados pré-natais. Tal normatização não apenas produz invisibilidade frente a possíveis identidades de gênero da pessoa gestante, mas compulsoriamente destina a esta "mulher", todo o foco de responsabilidades em torno da saúde da criança 56.

Neste estudo, tal dinâmica se fez presente ao se perceber que enquanto a maioria dos usuários da triagem auditiva neonatal são filhos de mulheres/responsáveis que realizaram testes para diagnóstico da infecção pelo HIV e triagem de sífilis, nenhum de seus "parceiros" recebeu a solicitação para a triagem de sífilis. Este dado acende um sinal de alerta para a necessidade de urgente sensibilização das parcerias sexuais de gestantes na detecção de infecções sexualmente transmissíveis, no âmbito da atenção básica. Em particular, durante o curso da gravidez, a fim de evitar casos de transmissão vertical e reinfecção por sífilis 57 . A magnitude de intervenções nesta área promoveria benefícios amplos e resultariam em potencial redução da carga de perda auditiva congênita atribuível a infecções durante o período gestacional 5 .

Nesta investigação, 46,11\% dos domicílios estava cadastrado na unidade de saúde da família ( $\mathrm{n}=$ 106.098). Mas esta característica não esteve significativamente associada à utilização da triagem auditiva neonatal. Um estudo conduzido por Sabbag \& Lacerda 58 na cidade de Curitiba, Paraná, entre 2013 e 2014, indicou que a unidade de saúde da família pode contribuir para o rastreamento e monitoramento das crianças assistidas por programas de triagem auditiva neonatal universal. Ao mesmo tempo em que nossos achados contrastam com a literatura mencionada, observa-se novamente a grande heterogeneidade existente no país. Ainda assim, a experiência curitibana exemplifica como diferentes estratégias de atenção básica poderiam se integrar no território em benefício da população usuária.

Em 2012, no Município de Jundiaí, no Estado de São Paulo, Campos et al. 59 observaram que apesar de médicos neonatologistas, pediatras e residentes em pediatria, serem os primeiros profissionais em contato com recém-nascidos e terem conhecimentos sobre fatores de risco para a detecção da perda auditiva congênita ou adquirida no período neonatal, cerca de 68,1\% afirmaram desconhecer quais seriam as técnicas específicas de avaliação da audição em neonatos. Logo, o desconhecimento sobre a triagem auditiva neonatal dentre os profissionais de saúde também revela-se como importante barreira a ser transposta 60,61.

Atividades de educação em saúde auditiva ainda não são amplamente adotadas em programas de triagem auditiva neonatal universal no Brasil 62. Porém, sua aplicabilidade pode ser um caminho profícuo ao tornarem as ações de promoção em saúde horizontais, na medida em que valorizam a posição do indivíduo enquanto responsável e protagonista de suas escolhas no processo saúde-doença-cuidado 63.

Neste sentido, Qirjazi et al. 64 destacam que o recebimento de informações acerca da triagem auditiva neonatal pode provocar ações positivas em relação à tomada de consciência acerca da saúde auditiva na infância. Ressaltam ainda que estas estratégias precisam ter especial atenção para residentes de áreas rurais e pessoas com piores condições socioeducacionais por majoritariamente serem grupos menos informados.

A análise do modo como a triagem auditiva neonatal foi utilizada nas capitais brasileiras sugere identidade com o perfil que Andersen \& Davidson 26 classificam como acesso desigual, visto que características individuais predisponentes sociodemográficas suplantaram aspectos de necessidade, direcionando quem seria beneficiado. Reformas nas dimensões contextuais de financiamento e organização impactariam substancialmente esta conformação a fim de redirecionar os serviços para que estes alcancem àqueles que mais demandam. Dado que o objeto em evidência é um serviço universal, compreende-se que acesso justo é aquele que promove acesso equitativo ao sistema de cuidados em saúde, para garantir justiça social.

Encontra-se em curso a implantação do Sistema Nacional de Triagem Neonatal (SISNEO), sistema de informação específico para a triagem neonatal integrada. Um de seus objetivos é incluir as triagens auditiva e ocular. Adicionalmente, se prevê a criação do SISNEO Centralizador, que armazenará dados em âmbitos nacional, estadual e municipal 65. 
Considerando potenciais encadeamentos, bem como a abrangência de suas implicações, avalia-se que este estudo oferece caminhos em direção aos objetivos e metas de desenvolvimento sustentável da Agenda 2030 publicados pela Organização das Nações Unidas (ONU), em 2015. Destes, destacamos o empenho global e coletivo em favor da erradicação da pobreza; saúde e bem-estar; educação de qualidade; igualdade de gênero; redução das desigualdades; paz, justiça e instituições eficazes 66 .

\section{Limitações}

Sub-representação de mães/responsáveis com perda auditiva, subgrupo que poderia ter maior percepção acerca da necessidade de utilização da triagem auditiva neonatal, configurando possível viés de seleção. Por outro lado, verifica-se que a informação sobre o desfecho foi condicionada à recordação do uso da triagem auditiva neonatal, não descartando-se viés de memória.

Dentre possíveis fontes de viés de informação lista-se: possíveis diferenças na aplicação dos questionários pela abrangência da PNS; Ausência de informações sobre a qualificação dos profissionais que realizaram a triagem auditiva neonatal, visto que apenas médicos e fonoaudiólogos são habilitados para realizar triagem auditiva; Dados indisponíveis para afirmar se todos os hospitais/maternidades em que as crianças nasceram ofereciam triagem auditiva; Indicadores contextuais provenientes de sistemas públicos de informação, cuja qualidade é discutível.

Finalmente, frisamos que as informações da PNS são autorreferidas. Portanto, não se desconsidera a possibilidade de haver discrepâncias entre os resultados deste tipo de delineamento e o que se teria, caso houvesse uso de procedimentos diagnósticos padronizados. Porém, a PNS é um estudo de base populacional e, na ausência de mensurações objetivas, as informações coletadas constituem uma opção adequada para a investigação sob estudo.

\section{Considerações finais}

Neste estudo, a proporção de extrema pobreza e o indicador de cobertura mensal da triagem auditiva neonatal destacaram-se como condições populacionais que influenciaram o uso da triagem auditiva neonatal. E faixa etária, raça/cor, escolaridade e atendimento pré-natal preponderantemente privado atuaram como determinantes socioeconômicos individuais.

Intersecções entre pobreza, pertencimento a subgrupos historicamente marginalizados e desigualdades geram não somente aquisição prejudicada de bens materiais, mas refletem preponderantemente em estados de saúde geral desfavoráveis. Estes elementos limitam o acesso a medidas de cuidado e educação em saúde, impactam em ausência de oportunidades, e por fim, resultam em representatividade desproporcional e falta de protagonismo perante decisões de instituições do Estado e da sociedade.

Estes condicionantes precisam ser superados para suscitar a promoção de ações que diminuam obstáculos perante a adoção de medidas no âmbito da saúde coletiva, como a triagem auditiva. Para isso, se faz necessário intervir em diferentes níveis. No domínio contextual, tendo como base a aplicação das leis e políticas relacionadas à saúde auditiva, já existentes, faz-se necessária a regulamentação da triagem auditiva neonatal. Maior valorização e capacitação dos profissionais da assistência, assim como atuação incisiva dos conselhos de classe profissionais, conjugados à participação social em busca da garantia de atenção integral à saúde neonatal. Depreende-se ainda que um sistema de informação específico, como o SISNEO Centralizador, contribuiria objetivamente para a melhor tomada de decisões envolvendo os setores de financiamento e organização dos serviços, além de viabilizar avanços na produção de conhecimento no Brasil.

$\mathrm{Na}$ dimensão individual, propõe-se focalizar neonatos em situação de vulnerabilidade, dentre as atividades contempladas pela atenção básica, assim como acolhimento multiprofissional a gestantes durante os cuidados pré-natais, pelo seu potencial em promover educação em saúde e autonomia, de modo a construir espaços para a partilha de vivências e conhecimentos que agreguem qualidade e eficácia aos serviços. 


\section{Colaboradores}

V. Melo-Ferreira contribuiu substancialmente para concepção e planejamento do estudo, construção do banco de dados e análise, interpretação dos resultados e redação do manuscrito. W. L. Junger contribuiu substancialmente para construção do banco de dados, metodologia de análise dos dados, interpretação dos resultados e revisão do manuscrito. G. L. Werneck contribuiu substancialmente para concepção, planejamento, análise, interpretação dos resultados, redação final do manuscrito e revisão crítica do conteúdo. Todos os autores aprovaram a versão final para publicação.

\section{Informações adicionais}

ORCID: Vanessa de Melo-Ferreira (0000-00027308-0676); Washington Leite Junger (0000-00026394-6587); Guilherme Loureiro Werneck (00000003-1169-1436)

\section{Agradecimentos}

À Coordenação de Aperfeiçoamento de Pessoal de Nível Superior (CAPES) e a Fundação Carlos Chagas Filho de Amparo à Pesquisa do Estado do Rio de Janeiro (FAPERJ) pelas bolsas de estudo em nível de Mestrado (Mestrado Nota 10). A todas as pessoas envolvidas na realização da Pesquisa Nacional de Saúde, 2013. Aos estudantes, trabalhadores e professores da Universidade do Estado do Rio de Janeiro (UERJ), por fazerem desta instituição uma potência constante.

\section{Referências}

1. Azevedo M, Pereira L, Vilanova L, Goulart A. Avaliação do processamento auditivo central: identificação de crianças de risco para alteração de linguagem e aprendizado durante o primeiro ano de vida. In: Marchesan IQ, Bolaffi C, organizadores. Tópicos em fonoaudiologia. São Paulo: Lovise; 1995. p. 447-62.

2. Hearing loss: an important global health concern. Lancet 2016; 387:2351.

3. Mason A, Mason M. Psychologic impact of deafness on the child and adolescent. Prim Care 2007; 34:407-26.

4. Kotby MN, Tawfik S, Aziz A, Taha H. Public health impact of hearing impairment and disability. Folia Phoniatr Logop 2008; 60:58-63.

5. Olusanya BO, Neumann KJ, Saunders JE. The global burden of disabling hearing impairment: a call to action. Bull World Health Organ 2014; 92:367-73.

6. World Health Organization. Deafness and hearing loss. http://www.who.int/news$\mathrm{room} /$ fact-sheets/detail/deafness-and-hear ing-loss (acessado em 07/Out/2020).

7. Harris MS, Dodson EE. Hearing health access in developing countries. Curr Opin Otolaryngol Head Neck Surg 2017; 25:353-8.

8. Wilson BS, Tucci DL, Merson MH, O'Donoghue GM. Global hearing health care: new findings and perspectives. Lancet 2017; 390:2503-15

9. Olusanya BO, Okolo AA. Adverse perinatal conditions in hearing-impaired children in a developing country. Paediatr Perinat Epidemiol 2006; 20:366-71.

10. Olusanya BO. Societal impact of bilirubin-induced hearing impairment in resource-limited nations. Semin Fetal Neonatal Med 2015; 20:58-63.

11. Caroça C, Vicente V, Campelo P, Chasqueira M, Caria H, Silva S, et al. Rubella in Sub-Saharan Africa and sensorineural hearing loss: a case control study. BMC Public Health 2017; 17:146.

12. Musiime GM, Seale AC, Moxon SG, Lawn JE. Risk of gentamicin toxicity in neonates treated for possible severe bacterial infection in lowand middle-income countries: systematic review. Trop Med Int Health 2015; 20:1593-606.

13. Acar B, Ocak E, Acar M, Kocaöz D. Comparison of risk factors in newborn hearing screening in a developing country. Turk J Pediatr 2015; 57:334-8.

14. Ullah S, Aslamkhan M, Ali A, Idrees M. Causes of deafness in the Punjab region of Pakistan and the role of consanguinity. Public Health 2017; 145:93-5.

15. Erenberg A, Lemons J, Sia C, Trunkel D, Ziring P. Newborn and infant hearing loss: detection and intervention. American Academy of Pediatrics. Task Force on Newborn and Infant Hearing, 1998-1999. Pediatrics 1999; 103:527-30. 
16. Lewis DR, Marone SAM, Mendes BCA, Cruz OLM, Nóbrega M. Multiprofessional committee on auditory health: COMUSA. Braz J Otorhinolaryngol 2010; 76:121-8.

17. American Academy of Pediatrics; Joint Committee on Infant Hearing. Year 2007 Position statement: principles and guidelines for early hearing detection and intervention programs. Pediatrics 2007; 120:898-921.

18. Departamento de Ações Programáticas Estratégicas, Secretaria de Atenção à Saúde, Ministério da Saúde. Diretrizes de atenção da triagem auditiva neonatal. http://bvsms.saude. gov.br/bvs/publicacoes/diretrizes_atencao_ triagem_auditiva_neonatal.pdf (acessado em 26/Set/2017).

19. Brasil. Lei no $12.303,2$ de agosto de 2010. Dispõe sobre a obrigatoriedade de realização do exame denominado Emissões Otoacústicas Evocadas. Diário Oficial da União 2010; 3 ago.

20. Ministério da Saúde. Portaria no 650, de 5 de outubro de 2011. Diário Oficial da União 2011; 6 out.

21. Ministério da Saúde. Portaria no 1.459 , de 24 de junho de 2011. Institui a Rede Cegonha no âmbito do Sistema Único de Saúde - SUS. Diário Oficial da União 2011; 27 jun.

22. Travassos C, Martins M. Uma revisão sobre os conceitos de acesso e utilização de serviços de saúde. Cad Saúde Pública 2004; 20 Suppl 2:S190-8.

23. Assis MMA, Jesus WLA. Acesso aos serviços de saúde: abordagens, conceitos, políticas e modelo de análise. Ciênc Saúde Colet 2012; 17:2865-75

24. Pavão ALB, Coeli CM. Modelos teóricos do uso de serviços de saúde: conceitos e revisão. Cad Saúde Colet (Rio J.) 2008;16:471-82.

25. Babitsch B, Gohl D, von Lengerke T. Re-revisiting Andersen's Behavioral Model of Health Services Use: a systematic review of studies from 1998-2011. Psychosoc Med 2012; 9:Doc11.

26. Andersen RM, Davidson P. Improving access to care in America: individual and contextual indicators. In: Kominski GF, editor. Changing the US Health Care System: key issues in health services policy and management. San Francisco: Jossey-Bass; 2014. p. 3-31.

27. Chapchap MJ, Segre CM. Universal newborn hearing screening and transient evoked otoacoustic emission: new concepts in Brazil. Scand Audiol 2001; 30:33-6.

28. Botelho MSN, Silva VB, Arruda LS, Kuniyoshi IC, Oliveira LLR, Oliveira AS. Newborn hearing screening in the Limiar Clinic in Porto Velho - Rondônia. Braz J Otorhinolaryngol 2010; 76:605-10

29. Oliveira JS, Rodrigues LB, Aurélio FS, Silva VB. Risk factors and prevalence of newborn hearing loss in a private health care system of Porto Velho, Northern Brazil. Rev Paul Pediatr 2013; 31:299-305.
30. Paschoal MR, Cavalcanti HG, Ferreira MAF. Análise espacial e temporal da cobertura da triagem auditiva neonatal no Brasil (20082015). Ciênc Saúde Colet 2017; 22:3615-24.

31. Cruz LRL, Ferrite S. Cobertura estimada da triagem auditiva neonatal para usuários do Sistema Único de Saúde, Brasil, 2008-2011. Rev Bras Saúde Mater Infant 2014; 14:401-11.

32. Silva LSG, Gonçalves CGO, Soares VMN, Silva LSG, Gonçalves CGO, Soares VMN. National Policy on Health Care Hearing: an evaluative study from covering services and diagnostic procedures. CoDAS 2014; 26:241-7.

33. Szwarcwald CL, Malta DC, Pereira CA, Vieira MLFP, Conde WL, Souza Júnior PRB de, et al. Pesquisa Nacional de Saúde no Brasil: concepção e metodologia de aplicação. Ciênc Saúde Colet 2014; 19:333-42.

34. Instituto Brasileiro de Geografia e Estatística. Pesquisa Nacional de Saúde, 2013: ciclos de vida. Brasil e grandes regiões. https://biblioteca. ibge.gov.br/visualizacao/livros/liv94522.pdf (acessado em 07/Out/2020).

35. Instituto Brasileiro de Geografia e Estatística. Pesquisa Nacional de Saúde. Microdados 2019. https://www.ibge.gov.br/estatisticasnovoportal/sociais/saude/9160-pesquisa-\%20 nacional-de-saude.html $?=\& \mathrm{t}=$ microdados (acessado em 07/Out/2020).

36. Atlas Brasil. Atlas do Desenvolvimento Humano no Brasil, 2019. http://atlasbrasil.org.br/ (acessado em 07/Out/2020).

37. Departamento de Informática do SUS. Nota Técnica dos indicadores municipais do rol de diretrizes, objetivos, metas e indicadores 2013-2015. http://tabnet.datasus.gov.br/cgi/ pacto/2015/Nota_Tecnica_Indicadores_Mu nicipais.pdf (acessado em 07/Out/2020).

38. Asparouhov T. General multi-level modeling with sampling weights. Commun Stat Theory Methods 2006; 35:439-60.

39. Lüdecke D. Package 'sjstats': collection of convenient functions for common statistical computations. https://cran.r-project.org/web/ packages/sjstats/sjstats.pdf (acessado em 07/ Out/2020).

40. Carle AC. Fitting multilevel models in complex survey data with design weights: Recommendations. BMC Med Res Methodol 2009; 9:49.

41. Twisk JWR. Applied multilevel analysis: a practical guide (Practical Guides to Biostatistics and Epidemiology). Cambridge: Cambridge University Press; 2006.

42. Kassambara A. Multicollinearity essentials and VIF in $\mathrm{R}$ - articles - STHDA. http://www.sthda.com/english/articles/39-regression-modeldiagnostics/160-multicollinearity-essentialsand-vif-in-r/ (acessado em 07/Out/2020).

43. Hartig F. DHARMa: residual diagnostics for hierarchical (multi-level/mixed) regression models. R package version 0.2.6. https:// CRAN.R-project.org/package $=$ DHARMa (acessado em 07/Out/2020). 
44. Rasbash J, Steele F, Browne WJ, Goldstein H. A user's guide to MLwiN. v. 3.03. http://www. bristol.ac.uk/cmm/media/software/mlwin/ downloads/manuals/3-03/manual-web.pdf (acessado em 07/Out/2020).

45. Melo-Ferreira V. Repositório TAN. GitHub. https://github.com/Melo-Ferreira/TAN (acessado em 07/Out/2020).

46. Malheiros MASF, Cavalcanti HG. Characterization of newborn hearing screening programs of maternity units located in the city of João Pessoa, PB, Brazil. Rev CEFAC 2015; 17:454-60.

47. Lima MLLT, Assis ABR, Mercês GB, Barros PF, Griz SMS. Socio-economic characteristic of mothers of a NHS program. Rev CEFAC 2008; 10:254-60.

48. Griz SMS, Barbosa CP, Silva ARA, Ribeiro MA, Menezes DC. Aspectos demográficos e socioeconômicos de mães atendidas em um programa de triagem auditiva neonatal. Rev Soc Bras Fonoaudiol 2010; 15:179-83.

49. Mallmann MB, Tomasi YT, Boing AF. Neonatal screening tests in Brazil: prevalence rates and regional and socioeconomic inequalities. J Pediatr (Rio J.) 2019; 96:487-94.

50. Bush ML, Kaufman MR, McNulty BN. Disparities in access to pediatric hearing health care. Curr Opin Otolaryngol Head Neck Surg 2017; 25:359-64.

51. Zeitlin W, Auerbach C, Mason SE, Spivak LG, Reiter B. Factors related to not following up with recommended testing in the diagnosis of newborn hearing loss. Health Soc Work 2017; 42:24-31.

52. Leal MC, Gama SGN, Cunha CB. Desigualdades raciais, sociodemográficas e na assistência ao pré-natal e ao parto, 1999-2001. Rev Saúde Pública 2005; 39:100-7.

53. Kalckmann S, Santos CG, Batista LE, Cruz VM. Racismo institucional: um desafio para a eqüidade no SUS? Saúde Soc 2007; 16:146-55.

54. Hogan VK, Araujo EM, Caldwell KL, Gonzalez-Nahm SN, Black KZ. "We black women have to kill a lion everyday": an intersectional analysis of racism and social determinants of health in Brazil. Soc Sci Med 2018; 199:96-105.

55. Ravi R, Gunjawate DR, Yerraguntla K, Lewis LE, Driscoll C, Rajashekhar B. Follow-up in newborn hearing screening - a systematic review. Int J Pediatr Otorhinolaryngol 2016; 90:29-36.
56. Pinho AR, Rodrigues L, Nogueira C. (Des) Construção da parentalidade trans: homens que engravidam. ex aequo 2020; (41):195-205.

57. Ministério da Saúde. Portaria no 77, 12 de janeiro de 2012. Dispõe sobre a realização de testes rápidos, na atenção básica, para a detecção de HIV e sífilis, assim como testes rápidos para outros agravos, no âmbito da atenção prénatal para gestantes e suas parcerias sexuais. Diário Oficial da União 2012; 13 jan.

58. Sabbag JC, Lacerda ABM. Rastreamento e monitoramento da Triagem Auditiva Neonatal em Unidade de Estratégia de Saúde da Família: estudo-piloto. CoDAS 2017; 29:e20160102.

59. Campos ACM, Shirane HY, Takemoto PVA, Lourenço EA. Universal newborn hearing screening: knowledge of pediatricians and neonatologists in the city of Jundiaí, São Paulo, Brazil. Braz J Otorhinolaryngol 2014; 80:379-85.

60. Soares CP, Marques LR, Flores NGC. Neonatal hearing screening: clinical applicability in the routine of the neonatologists pediatrician doctors. Rev CEFAC 2008; 10:110-6.

61. Colozza P, Anastasio ART. Screening, diagnosing and treating deafness: the knowledge and conduct of doctors serving in neonatology and/or pediatrics in a tertiary teaching hospital. São Paulo Med J 2009; 127:61-5.

62. Silva ARA, Silveira AK, Curado NRPV, Muniz LF, Griz SMS. Acompanhamento em programas de saúde auditiva infantil: uma revisão integrativa. Rev CEFAC 2014; 16:992-1003.

63. Barbosa CP, Griz SMS. Educação em saúde com vistas à triagem neonatal e audição: uma revisão integrativa. Rev CEFAC 2014; 16:643-50.

64. Qirjazi B, Toçi E, Tushe E, Burazeri G, Roshi E. Mothers' perceptions of universal newborn hearing screening in transitional Albania. PLoS One 2020; 15:e0237815.

65. Ministério da Saúde. Reformulação do Programa Nacional de Triagem Neonatal (PNTN). http://www.saude.gov.br/component/content/ article/821-acoes-e-programas/programa-na cional-da-triagem-neonatal/40739-reformu lacao-do-pntn (acessado em 07/Out/2020).

66. Organização das Nações Unidas. Agenda 2030. https://nacoesunidas.org/pos2015/agenda 2030/ (acessado em 07/Out/2020). 
Abstract

The current study assessed the effect of contextual and individual determinants of the use of newborn hearing screening in Brazilian state capitals and the Federal District. The conceptual theoretical model proposed by Andersen \&s Davidson (2014) oriented the analyses using multilevel logistic modeling with data from the Brazilian National Health Survey, 2013. The study population $(n=$ 585 ) is representative of 230,112 pairs of women/ responsible person over 18 years of age and their respective children under 2 years of age. At the contextual level (state capitals and Federal District), the use of newborn hearing screening was determined by the proportion of extremely poor individuals (odds ratio $-O R=0.91 ; 95 \%$ confidence interval - 95\%CI: 0.83-0.99) and by monthly coverage of newborn hearing screening $(O R=1.02$; 95\%CI: 1.01-1.02). At the individual level, use of newborn hearing screening was higher in the maternal age bracket 25 to 39 years, compared to $<25$ years. The odds of use of newborn hearing screening were lower in mothers with brown race/color $(\mathrm{OR}=0.47$; 95\%CI: 0.26-0.83) compared to white mothers. As for schooling, complete university education nearly tripled the odds of newborn hearing screening when compared to primary schooling $(O R=2.99 ; 95 \%$ CI: 1.15-7.79). Predominantly private prenatal care increased the odds of using newborn hearing screening by 2.18 times, compared to public prenatal care $(\mathrm{OR}=2.18 ; 95 \% \mathrm{CI}$ : 1.02-4.64). Effective enforcement of existing hearing health laws and policies and prioritization of primary healthcare and health education practices with a focus on more vulnerable newborns, based on the characteristics identified in this study, are initiatives that can help ensure an equitable social protection system.

Neonatal Screening; Hearing; Social Determinants of Health; Epidemiologic Models; Health Services Accessibility

\section{Resumen}

El presente estudio evaluó el efecto de determinantes contextuales e individuales en la utilización del tamizaje auditivo neonatal en el distrito federal y capitales brasileñas. El modelo teórico conceptual, propuesto por Andersen ef Davidson (2014), orientó los análisis mediante un modelado logístico multinivel, con datos de la Encuesta Nacional de Salud, 2013. La población observada $(n=585)$ es representativa de 230.112 parejas de mujeres/ responsables con más de 18 años y sus respectivos niños menores de 2 años de edad. En el nivel contextual (distrito federal y capitales), la utilización del tamizaje auditivo neonatal estuvo determinada por la proporción de individuos extremadamente pobres (oportunidad relativa $-O R=$ 0,91; intervalo de $95 \%$ de confianza - IC95\%: $0,83-0,99)$ y por la cobertura mensual del tamizaje auditivo neonatal (OR = 1,02; IC95\%: 1,011,02). En el nivel individual, se verificó un mayor uso de tamizaje auditivo neonatal en la franja etaria entre 25 a 39 años, en comparación con las de $<25$ años. La oportunidad de utilización de tamizaje auditivo neonatal fue menor en informantes con raza/color de la piel parda $(O R=$ 0,47; IC95\%: 0,26-0,83), en comparación con las blancas. Respecto a la escolaridad, se verificó que posee un Nivel Superior completo casi triplicó la oportunidad de uso de tamizaje auditivo neonatal, en contraste con el Nivel Fundamental de estudio formal (OR = 2,99; IC95\%: 1,15-7,79). El prenatal, predominantemente privado, aumentó en 2,18 veces la oportunidad de utilizar el tamizaje auditivo neonatal, en comparación con el público $(O R=$ 2,18; IC95\%: 1,02-4,64). La aplicación efectiva de leyes y politicas de salud auditiva ya existentes, $y$ la priorización de prácticas de atención básica y educación en salud, centrándose en neonatos de mayor vulnerabilidad, conforme las características identificadas en ese estudio, son iniciativas para exhibir un sistema de protección social ecuánime.

Tamizaje Neonatal; Audición; Determinantes Sociales de la Salud; Modelos Epidemiológicos; Accesibilidad a los Servicios de Salud

Recebido em 07/Out/2020

Versão final reapresentada em 31/Mar/2021

Aprovado em 29/Abr/2021 